\title{
Supplementary material for Spatial characterization of long-term hydrological change in the Arkavathy watershed adjacent to Bangalore, India
}

\section{Landsat imagery}

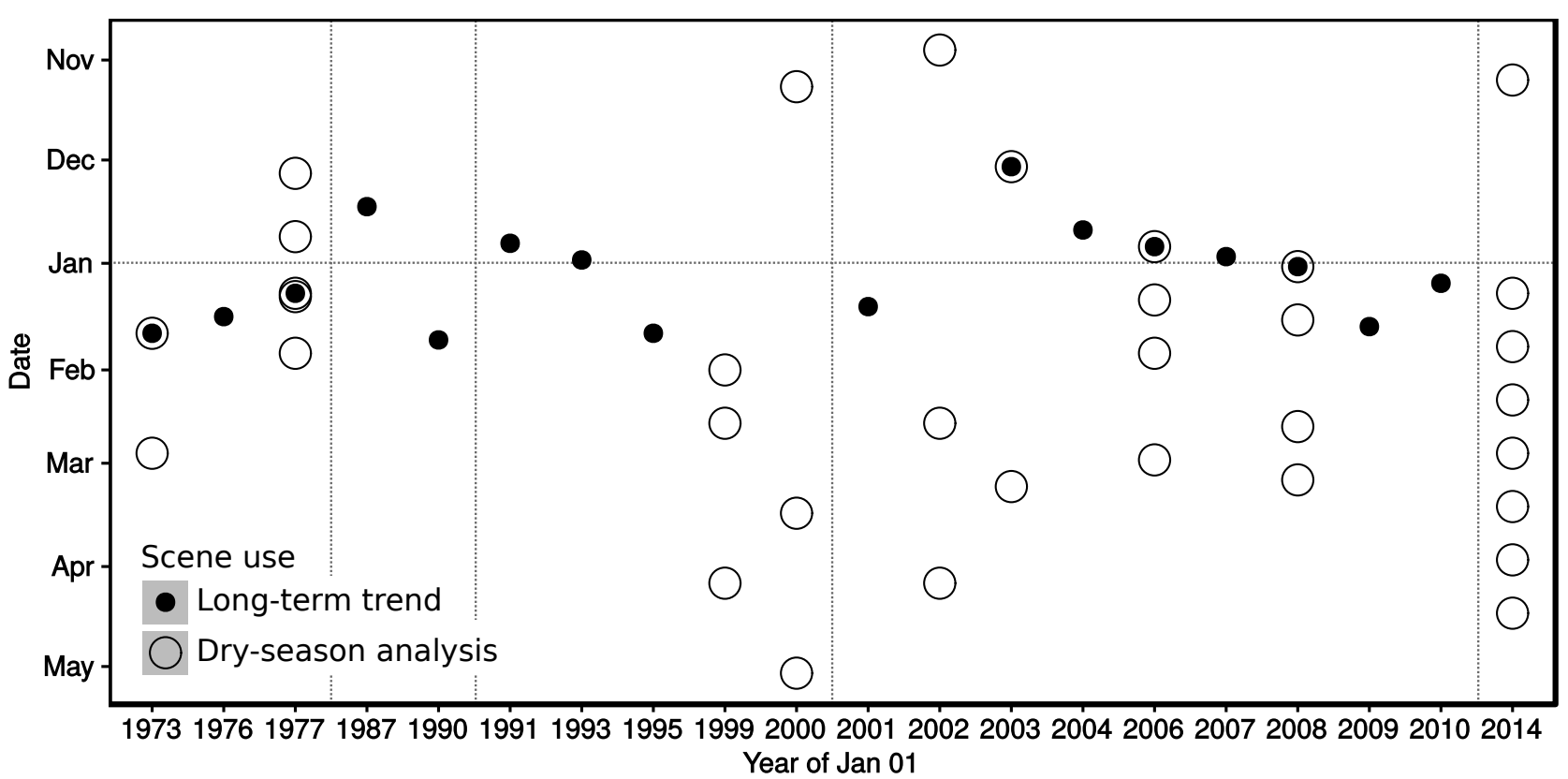

Figure 1: Landsat scenes analyzed in this study $(\mathrm{N}=45)$, with the year corresponding to the date on January 1. Decades are separated by dashed vertical lines using "monsoon year" (e.g., the January 1990 image is grouped with the 1980s because it corresponds to the 1989 monsoon year).

\begin{tabular}{llllllll}
\hline Date & Mission & Sensor & Path & Row & Cloud Free & Source & Use \\
\hline 1973 January 22 & Landsat 1 & MSS & 154 & 051 & Yes & USGS & LTT,DSA \\
1973 February 27 & Landsat 1 & MSS & 154 & 051 & Yes & USGS & DSA \\
1976 January 17 & Landsat 2 & MSS & 155 & 051 & Yes & USGS & LTT \\
1976 December 05 & Landsat 2 & MSS & 154 & 051 & & USGS & DSA \\
1976 December 24 & Landsat 2 & MSS & 155 & 051 & Yes & USGS & DSA \\
1977 January 10 & Landsat 2 & MSS & 154 & 051 & Yes & USGS & LTT,DSA \\
1977 January 11 & Landsat 2 & MSS & 155 & 051 & Yes & USGS & DSA \\
1977 January 28 & Landsat 2 & MSS & 154 & 051 & Yes & USGS & DSA \\
1986 December 15 & Landsat 5 & TM & 144 & 051 & Yes & NRSC & LTT \\
1990 January 24 & Landsat 5 & TM & 144 & 051 & Yes & NRSC & LTT \\
\hline
\end{tabular}

Continued on next page 


\begin{tabular}{|c|c|c|c|c|c|c|c|}
\hline Date & Mission & Sensor & Path & Row & Cloud Free & Source & Use \\
\hline 1990 December 26 & Landsat 5 & TM & 144 & 051 & & NRSC & LTT \\
\hline 1992 December 31 & Landsat 5 & $\mathrm{TM}$ & 144 & 051 & Yes & NRSC & LTT \\
\hline 1995 January 22 & Landsat 5 & $\mathrm{TM}$ & 144 & 051 & Yes & NRSC & LTT \\
\hline 1999 February 02 & Landsat 5 & $\mathrm{TM}$ & 144 & 051 & Yes & USGS & DSA \\
\hline 1999 February 18 & Landsat 5 & TM & 144 & 051 & Yes & USGS & DSA \\
\hline 1999 April 07 & Landsat 5 & $\mathrm{TM}$ & 144 & 051 & Yes & USGS & DSA \\
\hline 1999 November 09 & Landsat 7 & ETM+ & 144 & 051 & Yes & USGS & DSA \\
\hline 2000 March 16 & Landsat 7 & ETM+ & 144 & 051 & Yes & USGS & DSA \\
\hline 2000 May 03 & Landsat 7 & ETM+ & 144 & 051 & & USGS & DSA \\
\hline 2001 January 14 & Landsat 7 & ETM+ & 144 & 051 & Yes & USGS & LTT \\
\hline 2001 October 29 & Landsat 7 & ETM+ & 144 & 051 & & USGS & DSA \\
\hline 2002 February 18 & Landsat 7 & ETM+ & 144 & 051 & Yes & USGS & DSA \\
\hline 2002 April 07 & Landsat 7 & ETM+ & 144 & 051 & & USGS & DSA \\
\hline 2002 December 03 & Landsat 7 & ETM+ & 144 & 051 & Yes & USGS & LTT,DSA \\
\hline 2003 March 09 & Landsat 7 & ETM+ & 144 & 051 & Yes & USGS & DSA \\
\hline 2003 December 22 & Landsat 7 & ETM+ & 144 & 051 & Yes & USGS & LTT \\
\hline 2005 December 27 & Landsat 7 & ETM+ & 144 & 051 & & USGS & LTT,DSA \\
\hline 2006 January 12 & Landsat 7 & ETM+ & 144 & 051 & Yes & USGS & DSA \\
\hline 2006 January 28 & Landsat 7 & ETM+ & 144 & 051 & & USGS & DSA \\
\hline 2006 March 01 & Landsat 7 & ETM+ & 144 & 051 & & USGS & DSA \\
\hline 2006 December 30 & Landsat 7 & ETM+ & 144 & 051 & Yes & USGS & LTT \\
\hline 2008 January 02 & Landsat 7 & ETM+ & 144 & 051 & Yes & USGS & LTT,DSA \\
\hline 2008 January 18 & Landsat 7 & ETM+ & 144 & 051 & Yes & USGS & DSA \\
\hline 2008 February 19 & Landsat 7 & ETM+ & 144 & 051 & Yes & USGS & DSA \\
\hline 2008 March 06 & Landsat 7 & ETM+ & 144 & 051 & Yes & USGS & DSA \\
\hline 2009 January 20 & Landsat 7 & ETM+ & 144 & 051 & Yes & USGS & LTT \\
\hline 2010 January 07 & Landsat 7 & ETM+ & 144 & 051 & & USGS & LTT \\
\hline 2013 November 07 & Landsat 8 & OLI & 144 & 051 & & USGS & DSA \\
\hline 2014 January 10 & Landsat 8 & OLI & 144 & 051 & & USGS & DSA \\
\hline 2014 January 26 & Landsat 8 & OLI & 144 & 051 & Yes & USGS & DSA \\
\hline 2014 February 11 & Landsat 8 & OLI & 144 & 051 & Yes & USGS & DSA \\
\hline 2014 February 27 & Landsat 8 & OLI & 144 & 051 & & USGS & DSA,ACC \\
\hline 2014 March 15 & Landsat 8 & OLI & 144 & 051 & Yes & USGS & DSA \\
\hline 2014 March 31 & Landsat 8 & OLI & 144 & 051 & Yes & USGS & DSA \\
\hline 2014 April 16 & Landsat 8 & OLI & 144 & 051 & & USGS & DSA \\
\hline
\end{tabular}

Table 1: Landsat scenes analyzed. The path and row numbers refer to WRS-1 for Landsat 1-3 and WRS-2 for Landsat 5-8. "Use" column indicates whether the scene was used for calculation of long-term trends (LTT), dryseason analysis (DSA), or for accuracy assessment (ACC). 


\section{Classification flags}
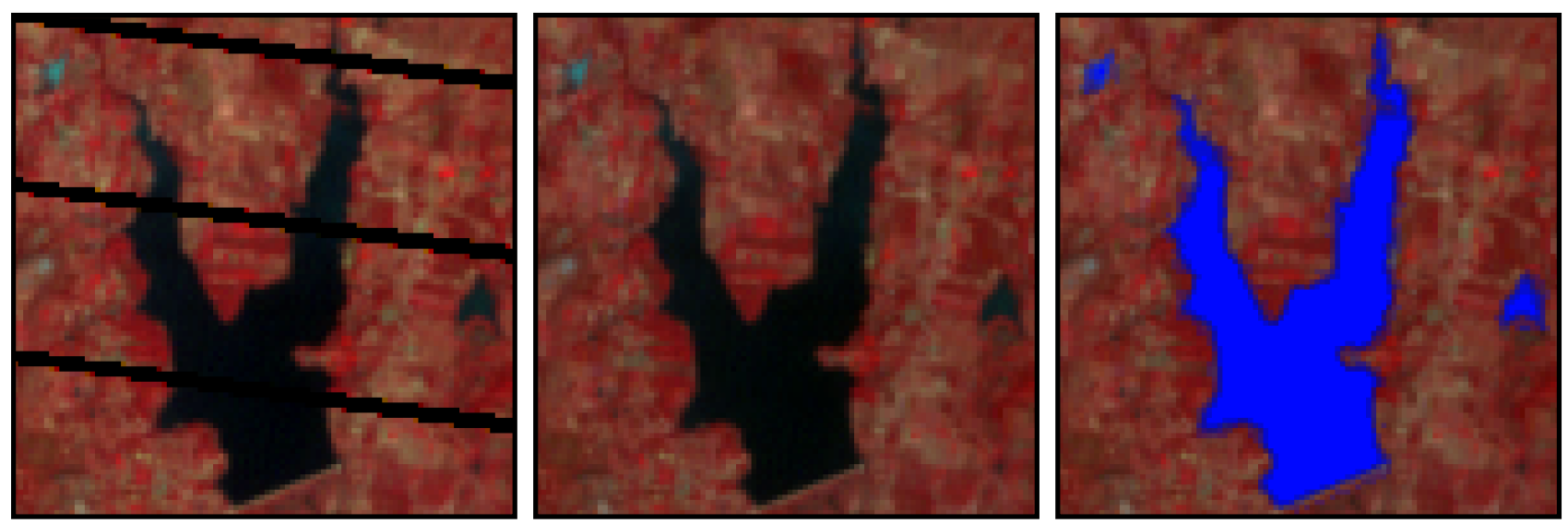

Figure 2: Left: Landsat false color composite (FCC) of a tank on 17 December 2005, with missing pixels visible as black diagonal bands. Middle: FCC after missing pixels were filled using successive grayscale dilation. Right: Classification of water in the image shown in blue.
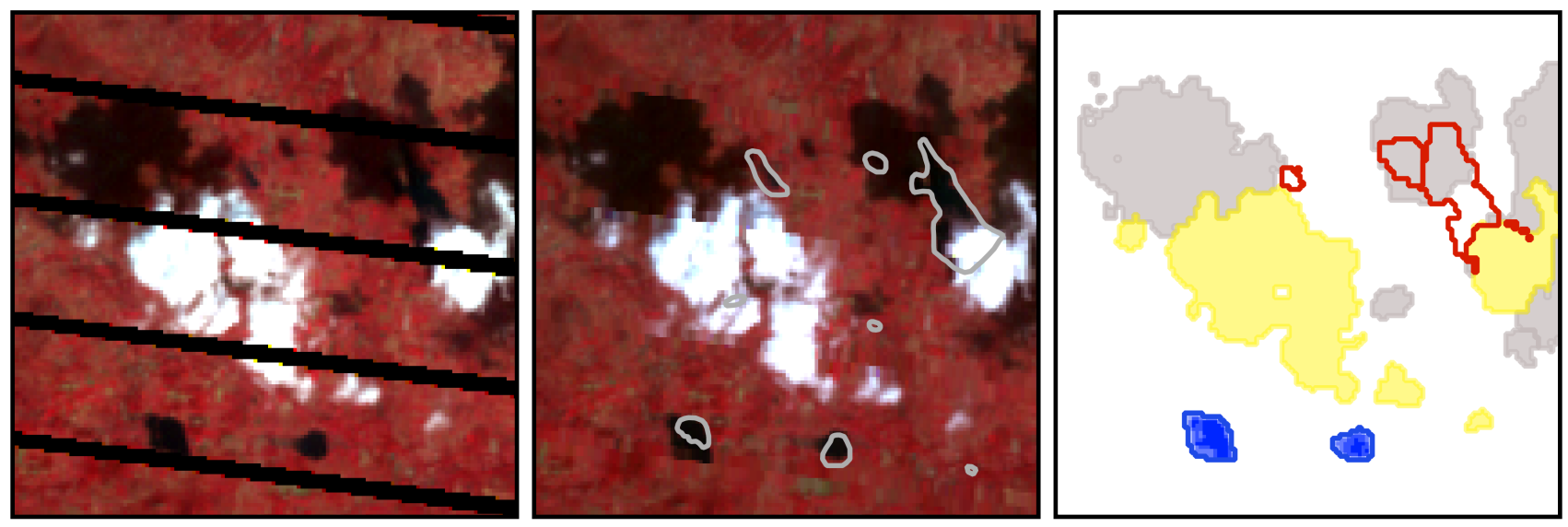

Figure 3: Left: Landsat false color composite on 27 December 2005 showing clouds and shadows near multiple tanks, with missing pixels visible as black diagonal bands. Middle: FCC after missing pixels were filled using successive grayscale dilation. Tank boundaries are shown in light gray. Right: Classification of clouds (yellow), cloud shadows (gray), water in tanks (blue), and areas classified as water but removed from the analysis due to clouds or cloud shadows (red outline). 


\section{Classification examples}
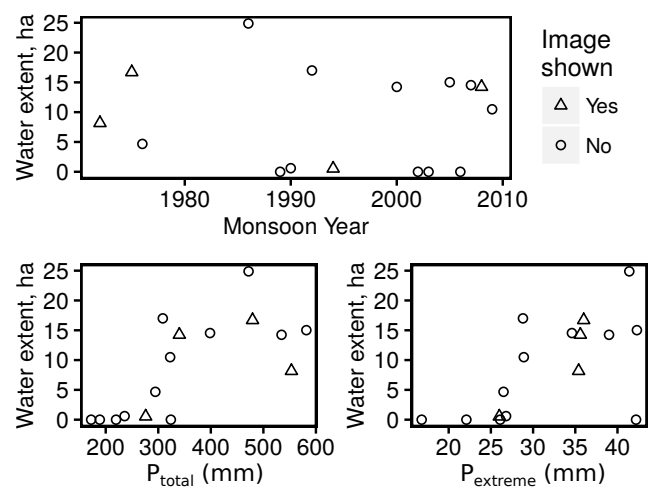

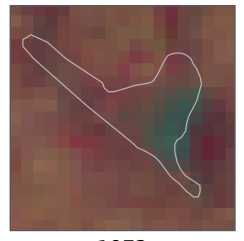

1972
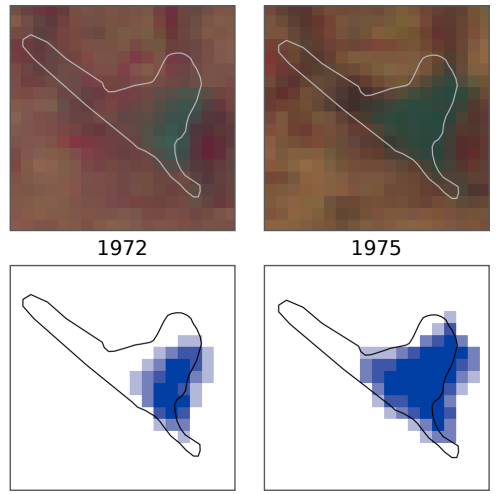

1975

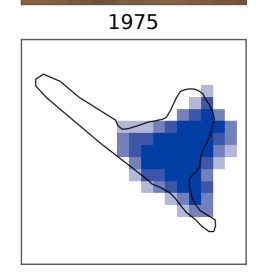

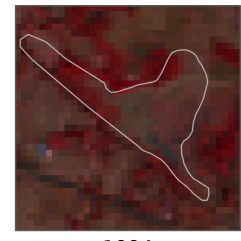

1994

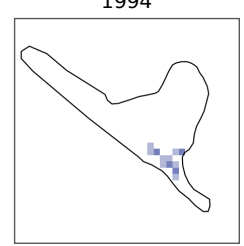

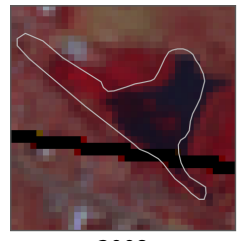

2008

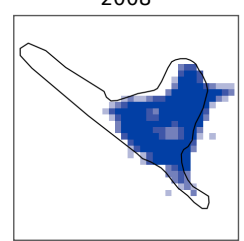

Figure 4: Left: Timeseries of tank water extent with selected Landsat images. Right: Landsat images (NIR-redgreen mapped to red-green-blue) and corresponding classified water fraction.
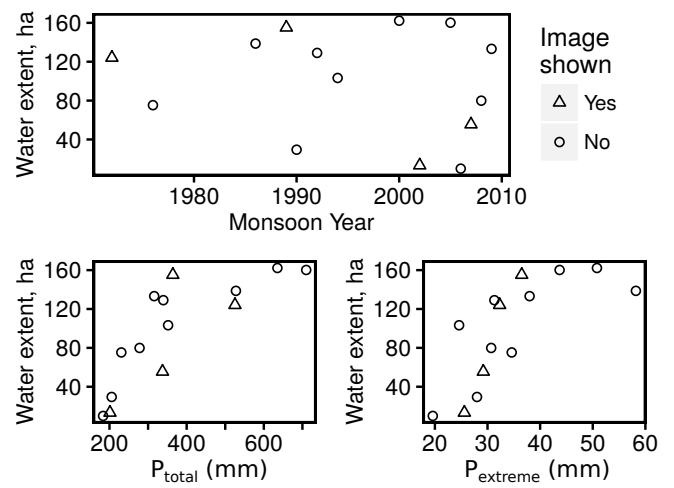

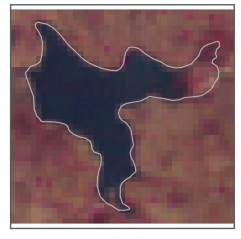

1972

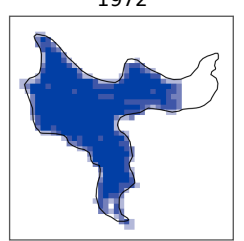

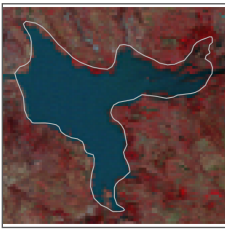

1986

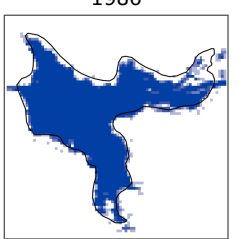

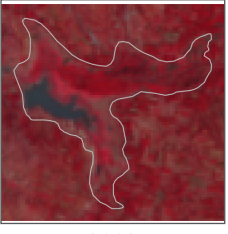

2002

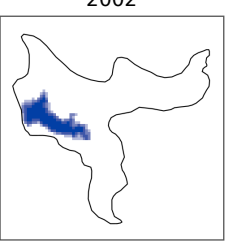

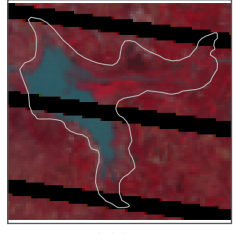

2007

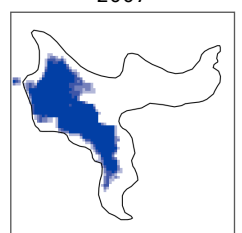

Figure 5: Left: Timeseries of tank water extent with selected Landsat images. Right: Landsat images (NIR-redgreen mapped to red-green-blue) and corresponding classified water fraction. 


\section{Results from multiple regression}

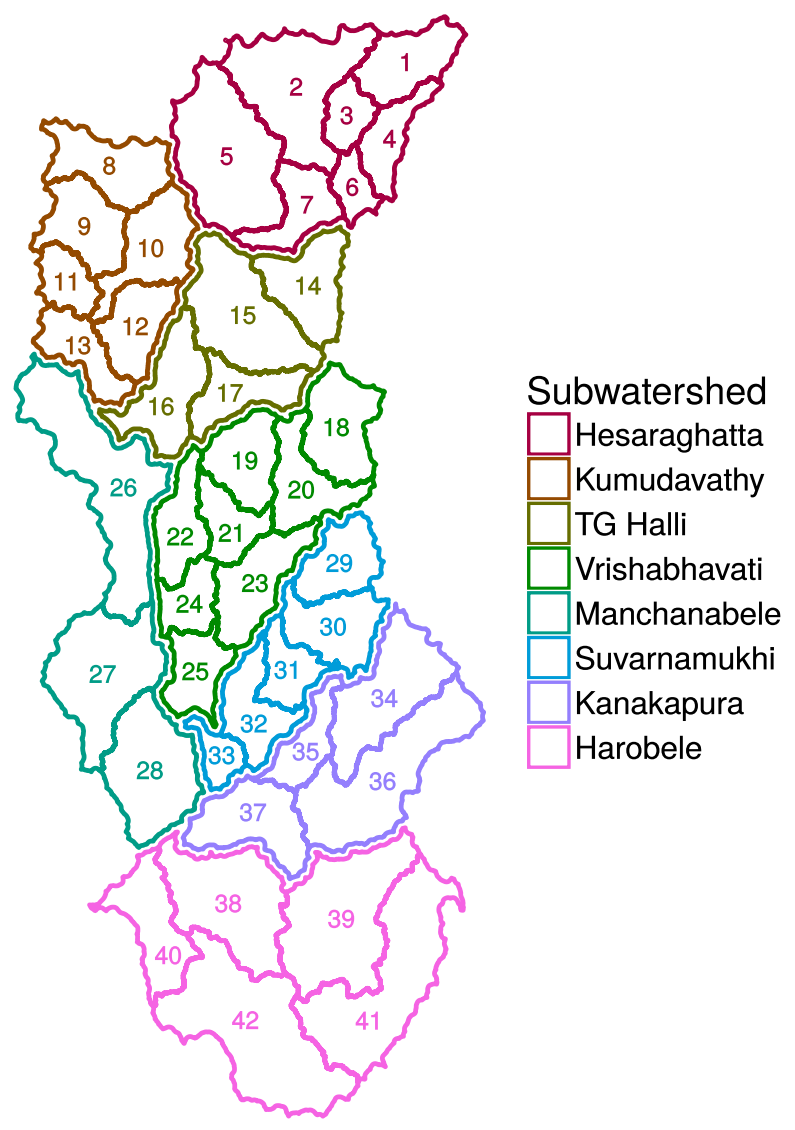

Figure 6: Subwatershed names and cluster IDs for the multiple regression. The Manchanabele and Harobele subwatersheds here are named for reservoirs within the watershed, which are not located at the subwatershed outlet. 


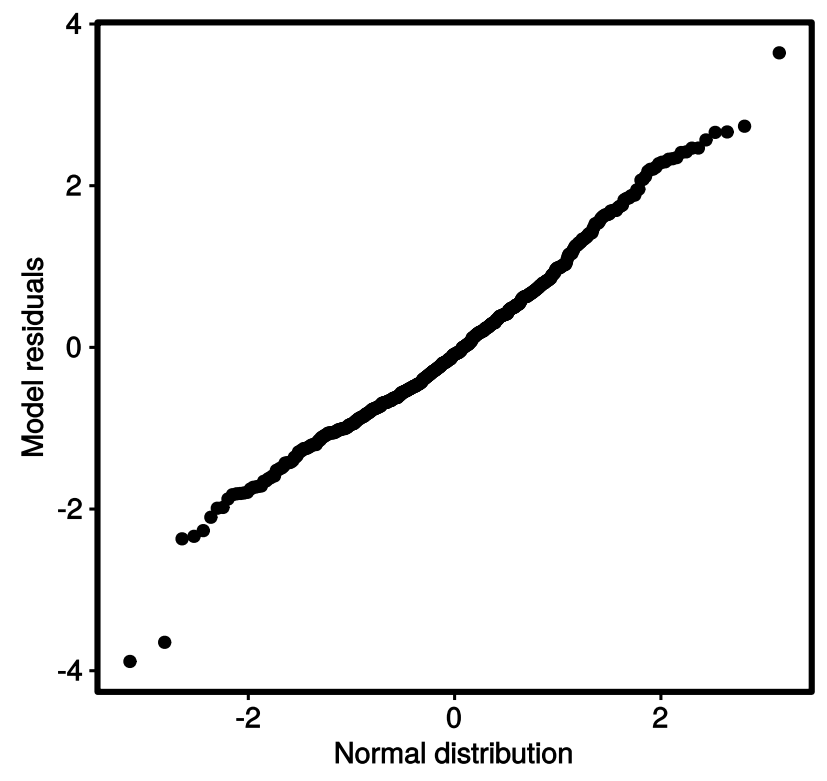

Figure 7: Quantile-quantile plot of residuals from multiple regression, with residuals normalized by mean and standard deviation and plotted agains a theoretical normal distribution.

\begin{tabular}{lllll}
\hline Variable & Watershed & Estimate & $95 \%$ Conf. Interval & Significant \\
\hline P_total & Hesaraghatta & 0.0030 & {$[0.0011,0.0048]$} & $\mathrm{Y}$ \\
P_total & Kumudavathy & 0.0048 & {$[0.0031,0.0065]$} & $\mathrm{Y}$ \\
P_total & TG Halli & 0.0053 & {$[0.0032,0.0073]$} & $\mathrm{Y}$ \\
P_total & Vrishabhavati & 0.0026 & {$[0.0013,0.0040]$} & $\mathrm{Y}$ \\
P_total & Manchanabele & 0.0052 & {$[0.0031,0.0074]$} & $\mathrm{Y}$ \\
P_total & Suvarnamukhi & 0.0029 & {$[0.0011,0.0048]$} & $\mathrm{Y}$ \\
P_total & Kanakapura & 0.0027 & {$[0.0007,0.0048]$} & $\mathrm{Y}$ \\
P_total & Harobele & 0.0037 & {$[0.0018,0.0057]$} & $\mathrm{Y}$ \\
P_extreme & Hesaraghatta & 0.0824 & {$[0.0192,0.1456]$} & $\mathrm{Y}$ \\
P_extreme & Kumudavathy & 0.0635 & {$[0.0065,0.1206]$} & $\mathrm{Y}$ \\
P_extreme & TG Halli & 0.0563 & {$[-0.0117,0.1243]$} & \\
P_extreme & Vrishabhavati & 0.0960 & {$[0.0509,0.1412]$} & $\mathrm{Y}$ \\
P_extreme & Manchanabele & 0.0345 & {$[-0.0293,0.0983]$} & \\
P_extreme & Suvarnamukhi & 0.0855 & {$[0.0277,0.1433]$} & $\mathrm{Y}$ \\
P_extreme & Kanakapura & 0.0728 & {$[0.0025,0.1431]$} & $\mathrm{Y}$ \\
P_extreme & Harobele & 0.0840 & {$[0.0295,0.1385]$} & $\mathrm{Y}$ \\
L_dryseason & Hesaraghatta & -0.0062 & {$[-0.0145,0.0021]$} & \\
L_dryseason & Kumudavathy & -0.0187 & {$[-0.0276,-0.0097]$} & $\mathrm{Y}$ \\
L_dryseason & TG Halli & -0.0169 & {$[-0.0277,-0.0062]$} & $\mathrm{Y}$ \\
L_dryseason & Vrishabhavati & -0.0042 & {$[-0.0119,0.0034]$} & \\
L_dryseason & Manchanabele & 0.0046 & {$[-0.0078,0.0170]$} & \\
L_dryseason & Suvarnamukhi & -0.0023 & {$[-0.0127,0.0080]$} & \\
L_dryseason & Kanakapura & 0.0081 & {$[-0.0033,0.0196]$} & \\
L_dryseason & Harobele & -0.0021 & {$[-0.0122,0.0080]$} & \\
Trend & Cluster 1 & -8.5423 & {$[-13.1524,-3.9321]$} & $\mathrm{Y}$ \\
Trend & Cluster 2 & -5.7428 & {$[-9.8688,-1.6168]$} & $\mathrm{Y}$ \\
\hline
\end{tabular}

Continued on next page 


\begin{tabular}{|c|c|c|c|c|}
\hline Variable & Watershed & Estimate & 95\% Conf. Interval & Significant \\
\hline Trend & Cluster 3 & -5.9023 & {$[-9.2785,-2.5262]$} & $\mathrm{Y}$ \\
\hline Trend & Cluster 4 & -6.0719 & [ -9.7290,-2.4149] & Y \\
\hline Trend & Cluster 5 & -3.0386 & {$[-6.3040,0.2268]$} & \\
\hline Trend & Cluster 6 & -4.9914 & {$[-7.5963,-2.3865]$} & $\mathrm{Y}$ \\
\hline Trend & Cluster 7 & -1.9311 & {$[-3.2717,-0.5905]$} & Y \\
\hline Trend & Cluster 8 & -6.0638 & {$[-9.8431,-2.2845]$} & $\mathrm{Y}$ \\
\hline Trend & Cluster 9 & -1.8920 & {$[-4.3096,0.5256]$} & \\
\hline Trend & Cluster 10 & -0.6443 & {$[-2.3368,1.0483]$} & \\
\hline Trend & Cluster 11 & -1.5497 & {$[-3.0651,-0.0344]$} & $\mathrm{Y}$ \\
\hline Trend & Cluster 12 & 0.5510 & {$[-0.3884,1.4904]$} & \\
\hline Trend & Cluster 13 & 0.2430 & {$[-0.4284,0.9144]$} & \\
\hline Trend & Cluster 14 & -2.4326 & {$[-3.8343,-1.0309]$} & Y \\
\hline Trend & Cluster 15 & -0.4811 & {$[-1.8790,0.9168]$} & \\
\hline Trend & Cluster 16 & -0.0440 & {$[-0.6246,0.5367]$} & \\
\hline Trend & Cluster 17 & 0.7534 & {$[-0.4355,1.9424]$} & \\
\hline Trend & Cluster 18 & -0.1927 & {$[-0.5592,0.1737]$} & \\
\hline Trend & Cluster 19 & 1.8104 & {$[0.1391,3.4817]$} & $\mathrm{Y}$ \\
\hline Trend & Cluster 20 & 1.3214 & {$[0.3053,2.3374]$} & $\mathrm{Y}$ \\
\hline Trend & Cluster 21 & 0.2823 & {$[-1.0812,1.6458]$} & \\
\hline Trend & Cluster 22 & 0.2253 & {$[-1.8224,2.2730]$} & \\
\hline Trend & Cluster 23 & 4.4629 & {$[1.3973,7.5285]$} & $\mathrm{Y}$ \\
\hline Trend & Cluster 24 & 0.3318 & {$[-0.5243,1.1879]$} & \\
\hline Trend & Cluster 25 & -0.4928 & {$[-1.6529,0.6673]$} & \\
\hline Trend & Cluster 26 & 0.0559 & {$[-0.1662,0.2779]$} & \\
\hline Trend & Cluster 27 & -0.0627 & {$[-0.5995,0.4741]$} & \\
\hline Trend & Cluster 28 & -0.5958 & {$[-1.7350,0.5433]$} & \\
\hline Trend & Cluster 29 & 2.1627 & {$[0.9886,3.3368]$} & $\mathrm{Y}$ \\
\hline Trend & Cluster 30 & 0.5555 & {$[-0.7622,1.8732]$} & \\
\hline Trend & Cluster 31 & 3.0785 & {$[-0.4827,6.6397]$} & \\
\hline Trend & Cluster 32 & -0.1133 & {$[-2.2537,2.0270]$} & \\
\hline Trend & Cluster 33 & 0.1548 & {$[-0.6438,0.9535]$} & \\
\hline Trend & Cluster 34 & 0.6434 & {$[-0.1912,1.4780]$} & \\
\hline Trend & Cluster 35 & -0.7273 & {$[-2.2420,0.7874]$} & \\
\hline Trend & Cluster 36 & 0.0639 & {$[-0.7680,0.8957]$} & \\
\hline Trend & Cluster 37 & -0.6639 & {$[-2.5395,1.2118]$} & \\
\hline Trend & Cluster 38 & -0.0057 & {$[-0.6712,0.6598]$} & \\
\hline Trend & Cluster 39 & 0.1250 & {$[-0.2911,0.5411]$} & \\
\hline Trend & Cluster 40 & -1.7551 & {$[-3.9551,0.4450]$} & \\
\hline Trend & Cluster 41 & -0.5841 & {$[-1.6055,0.4373]$} & \\
\hline Trend & Cluster 42 & 0.1273 & {$[-0.1511,0.4058]$} & \\
\hline
\end{tabular}

Table 2: Results from multiple regression for all varibles. The effects that apply at the subwatershed level are reported as directly output from the model. The temporal trend of each cluster was converted to units of ha per decade per 10 square km, matching Figure 8. 


\section{Dry-season analysis}
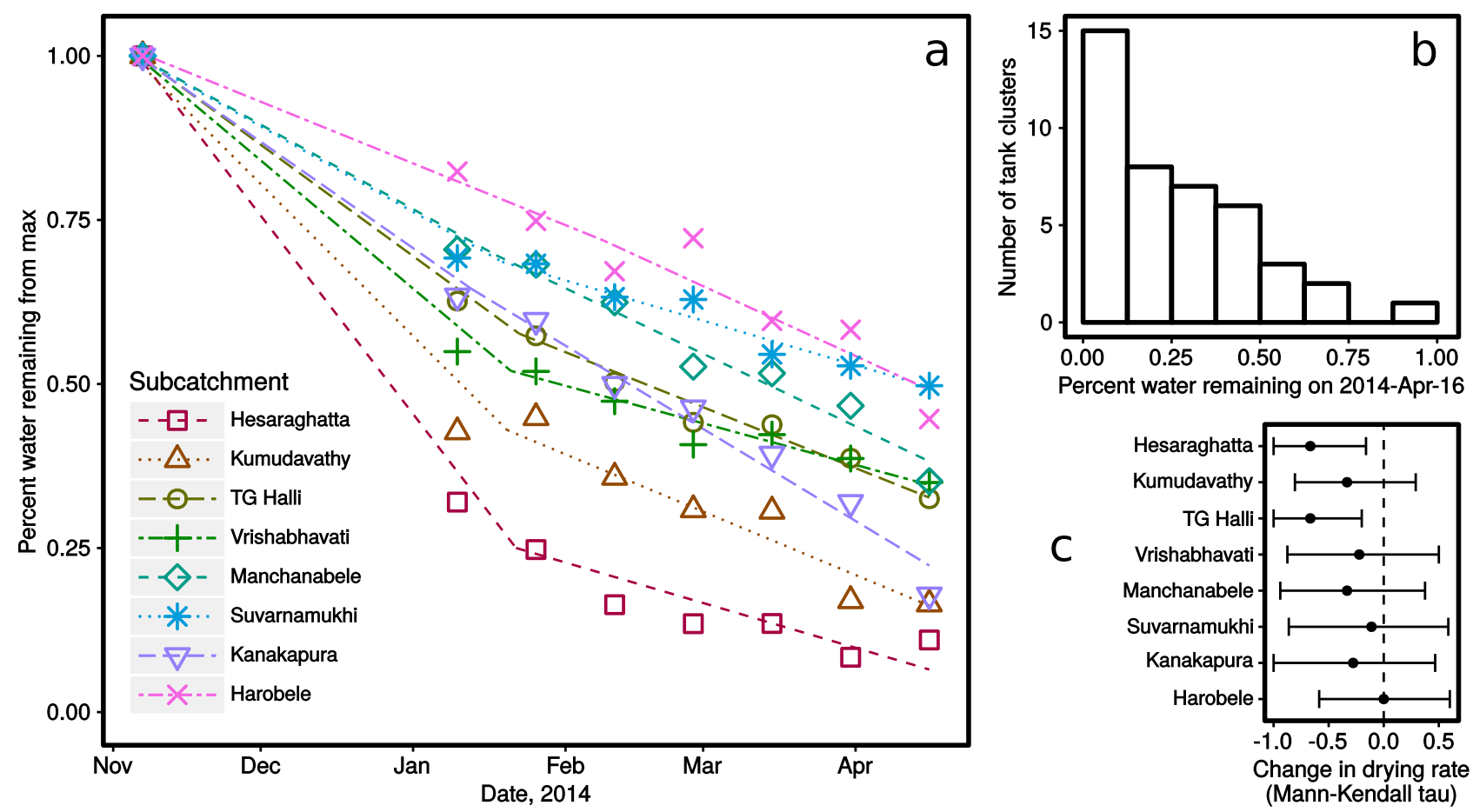

Figure 8: Dryseason analysis. (a) Post-monsoon drying of all tanks in each subwatershed, relative to the water extent at start of the dry season. The symbol for each subcatchment corresponds to the symbol in Figure 8 of Section 4.2 of the main article. (b) Histogram of the remaining water at the end of dry season as a fraction of the start of the dry season for all tank clusters. (c) Confidence intervals on the Mann-Kendall test statistic (tau) for a trend in the rate of tank water loss in dry season. Most subwatersheds do not exhibit a statistically significant trend in the rate of dry-season water loss (the confidence intervals include zero), but the Hesaraghatta and TG Halli subwatersheds exhibit a significant decreasing trend, meaning that tanks dry at a slower rate now than in the past. 